\title{
Dipole excitation and geometry of borromean nuclei
}

\author{
K. Hagino ${ }^{1}$ and H. Sagawa ${ }^{2}$ \\ 1 Department of Physics, Tohoku University, Sendai, 980-8578, Japan \\ ${ }^{2}$ Center for Mathematical Sciences, University of Aizu, Aizu-Wakamatsu, Fukushima 965-8560, Japan
}

\begin{abstract}
We analyze the Coulomb breakup cross sections of ${ }^{11} \mathrm{Li}$ and ${ }^{6} \mathrm{He}$ nuclei using a three-body model with a density-dependent contact interaction. We show that the concentration of the $\mathrm{B}(\mathrm{E} 1)$ strength near the threshold can be well reproduced with this model. With the help of the calculated B(E1) value, we extract the root-mean-square (rms) distance between the core nucleus and the center of mass of two valence neutrons without resorting to the sum rule, which may suffer from unphysical Pauli forbidden transitions. Together with the empirical rms distance between the neutrons obtained from the matter radius study and also from the three-body correlation study in the break-up reaction, we convert these rms distances to the mean opening angle between the valence neutrons from the core nucleus. We find that the obtained mean opening angles in ${ }^{11} \mathrm{Li}$ and ${ }^{6} \mathrm{He}$ agree with the three-body model predictions.
\end{abstract}

PACS numbers: 25.60.-t,21.45.+v,21.60.Gx,25.60.Gc

It has been well recognized by now that weakly bound nuclei exhibit a strong multipole strength which is concentrated near the continuum threshold, because of the optimal matching of wave functions between a weakly bound and continuum states [1, 2, 3, 4, 5]. Recently, Nakamura et al. have remeasured the low-lying dipole excitations in ${ }^{11} \mathrm{Li}$ nucleus and have confirmed for the first time the strong concentration of the dipole strength near the threshold in this 2-neutron (2n) halo nucleus [6]. The low-lying dipole strength for another $2 \mathrm{n}$ halo nucleus, ${ }^{6} \mathrm{He}$, has also been measured by Aumann et al. [7].

As well as being of considerable interest in its own right, the $\mathrm{B}(\mathrm{E} 1)$ strength distribution of 2-neutron halo nuclei is also important as it is intimately related to the root-mean-square (rms) distance, $\sqrt{\left\langle r_{c-2 n}^{2}\right\rangle}$, between the core nucleus and the center-of-mass of two valence neutrons [8, 9, 10]. Together with an additional information for the rms distance between the two neutrons, $\sqrt{\left\langle r_{n n}^{2}\right\rangle}$, one can then extract the geometry of $2 \mathrm{n}$ halo nuclei, such as the mean opening angle between the neutrons from the core [6, 11]. This information is particularly important to extract the strength of di-neutron correlations in halo nuclei.

In the previous applications, the rms distance $\sqrt{\left\langle r_{c-2 n}^{2}\right\rangle}$ has been obtained from the measured B(E1) strength using the relation [8, 9, 10],

$$
B(E 1)=\frac{3}{\pi}\left(\frac{Z e}{A}\right)^{2}\left\langle r_{c-2 n}^{2}\right\rangle .
$$

This relation is obtained with closure, which includes unphysical Pauli forbidden transitions to the states with negative excitation energies. Although the effect of Pauli forbidden transitions is not large, it leads to a nonnegligible correction. In Ref. [10], a better prescription has been proposed recently, which uses a model calculation for the $\mathrm{B}(\mathrm{E} 1)$ value and $\left\langle r_{c-2 n}^{2}\right\rangle$ to extract the "experimental value" for $\left\langle r_{c-2 n}^{2}\right\rangle$ (See Eq. (6) in Ref.
[10], and Eq. (21) below). Although this prescription uses theoretical values, it has been shown that the model dependence is insignificant [10].

The aim of this paper is to analyze the mean opening angle of valence neutrons in the $2 \mathrm{n}$ halo nuclei, ${ }^{11} \mathrm{Li}$ and ${ }^{6} \mathrm{He}$, using several empirical information. To this end, we first discuss the new prescription for $\left\langle r_{c-2 n}^{2}\right\rangle$ by analyzing the Coulomb dissociation cross sections of these nuclei with a three-body model. Assuming the three-body character, one can also extract the distance between two neutrons, $\sqrt{\left\langle r_{n n}^{2}\right\rangle}$, from the empirical information of matter radii and $\left\langle r_{c-2 n}^{2}\right\rangle$ (see Eq. (3)). An alternative way to extract the value for $\sqrt{\left\langle r_{n n}^{2}\right\rangle}$ is the three-body correlation study in the dissociation of two neutrons in halo nuclei [12. We will discuss the two ways to determine the mean opening angle by using these empirical information.

The three-body model which we employ in this paper is exactly the same as in Refs. [13, 14, 15]. The model adapts a density-dependent contact interaction between the valence neutrons $[8,9]$. The recoil kinetic energy of the core nucleus is taken into account as in Ref. 15]. Single particle continuum states are discretized by putting a nucleus in a large box. The wave functions for the ground state with $J^{\pi}=0^{+}$and for the excited $J^{\pi}=1^{-}$ states are then obtained by diagonalizing the three-body Hamiltonian within a large model space which is consistent with the $n n$ interaction. We use the same values for the parameters as in Ref. [13].

The dipole strength distributions for the ${ }^{6} \mathrm{He}$ and ${ }^{11} \mathrm{Li}$ nuclei obtained with this model are shown in Fig. 1 1. Also shown by the solid curves are the B(E1) distributions smeared with the Lorenzian function with the

1 We have found that the matrix elements for the off-diagonal part of the recoil kinetic energy were not properly evaluated for the $J^{\pi}=1^{-}$states in Fig. 5 of Ref. [13]. This technical problem has been cured in Fig. 1 of the present manuscript (it had been cured in the $\mathrm{B}(\mathrm{E} 2)$ calculations of ${ }^{16} \mathrm{C}$ in Ref. [16]). Although this error did not cause any substantial change in ${ }^{11} \mathrm{Li}$, the $\mathrm{B}(\mathrm{E} 1)$ 


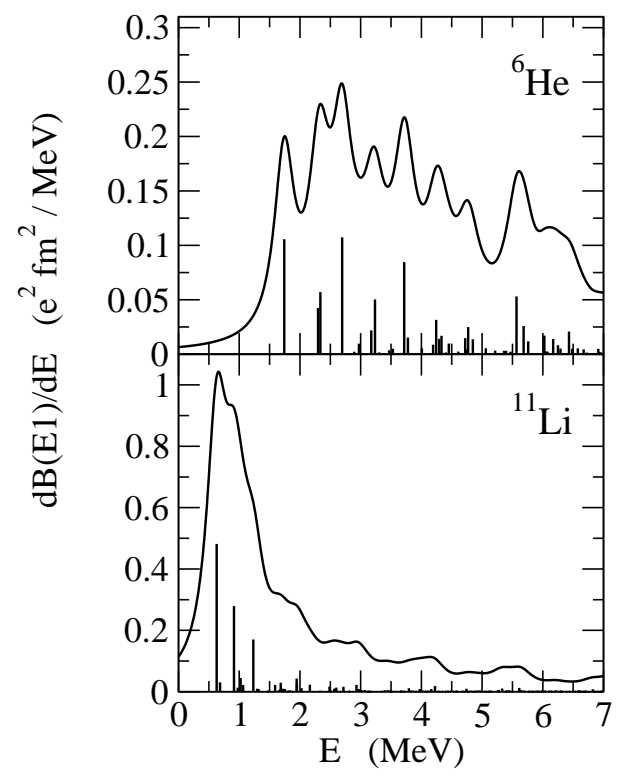

FIG. 1: The B(E1) distribution for the ${ }^{6} \mathrm{He}$ and ${ }^{11} \mathrm{Li}$ nuclei. The solid curve is obtained with a smearing procedure with $\Gamma=0.2 \mathrm{MeV}$.

width of $\Gamma=0.2 \mathrm{MeV}$. For the ${ }^{6} \mathrm{He}$ nucleus, we obtain the total $\mathrm{B}(\mathrm{E} 1)$ strength of $0.660 \mathrm{e}^{2} \mathrm{fm}^{2}$ up to $E \leq 5$ $\mathrm{MeV}$ and $1.053 \mathrm{e}^{2} \mathrm{fm}^{2}$ up to $E \leq 10 \mathrm{MeV}$. These are in good agreement with the experimental values, $\mathrm{B}$ (E1; $E \leq 5 \mathrm{MeV})=0.59 \pm 0.12 \mathrm{e}^{2} \mathrm{fm}^{2}$ and $\mathrm{B}(\mathrm{E} 1 ; E \leq 10$ $\mathrm{MeV})=1.2 \pm 0.2 \mathrm{e}^{2} \mathrm{fm}^{2}$ 7]. For the ${ }^{11} \mathrm{Li}$ nucleus, we obtain the total B(E1) strength of $1.405 \mathrm{e}^{2} \mathrm{fm}^{2}$ up to $E_{\text {rel }}=E-S_{2 n} \leq 3 \mathrm{MeV}$, which is compared to the experimental value, $\mathrm{B}\left(\mathrm{E} 1 ; E_{\text {rel }} \leq 3 \mathrm{MeV}\right)=1.42 \pm 0.18$ $\mathrm{e}^{2} \mathrm{fm}^{2}$ [6]. Again, the experimental data is well reproduced within the present model. In Ref. [10], it has been proposed to estimate the experimental value for $\left\langle r_{c-2 n}^{2}\right\rangle$ using the relation,

$$
\left\langle r_{c-2 n}^{2}\right\rangle_{\exp }=\frac{B\left(E 1 ; E \leq E_{\max }\right)_{\exp }}{B\left(E 1 ; E \leq E_{\max }\right)_{\mathrm{cal}}} \cdot\left\langle r_{c-2 n}^{2}\right\rangle_{\mathrm{cal}} .
$$

From the calculated values for $\left\langle r_{c-2 n}^{2}\right\rangle_{\text {cal }}$, that is, 13.2 and $26.3 \mathrm{fm}^{2}$ for ${ }^{6} \mathrm{He}$ and ${ }^{11} \mathrm{Li}$, respectively, we thus obtain $\sqrt{\left\langle r_{c-2 n}^{2}\right\rangle_{\exp }}=3.878 \pm 0.324 \mathrm{fm}$ and $5.15 \pm 0.327 \mathrm{fm}$ for ${ }^{6} \mathrm{He}$ and ${ }^{11} \mathrm{Li}$, respectively. Notice that the value for the ${ }^{6} \mathrm{He}$ nucleus is somewhat larger than the one estimated in Ref. [7], that is, $3.36 \pm 0.39 \mathrm{fm}$.

distribution for ${ }^{6} \mathrm{He}$ is considerably different from the previous calculation. The energy of the first peak in the $\mathrm{B}(\mathrm{E} 1)$ distribution is now at 1.75 and $0.66 \mathrm{MeV}$ for ${ }^{6} \mathrm{He}$ and ${ }^{11} \mathrm{Li}$, respectively (previously, it was at 1.55 and $0.66 \mathrm{MeV}$ for ${ }^{6} \mathrm{He}$ and ${ }^{11} \mathrm{Li}$, respectively). These values are still close to $1.6 S_{2 n}$, where $S_{2 n}$ is the $2 \mathrm{n}$ separation energy, and our conclusion that the dineutron correlation plays an important role in these nuclei remains unchanged.

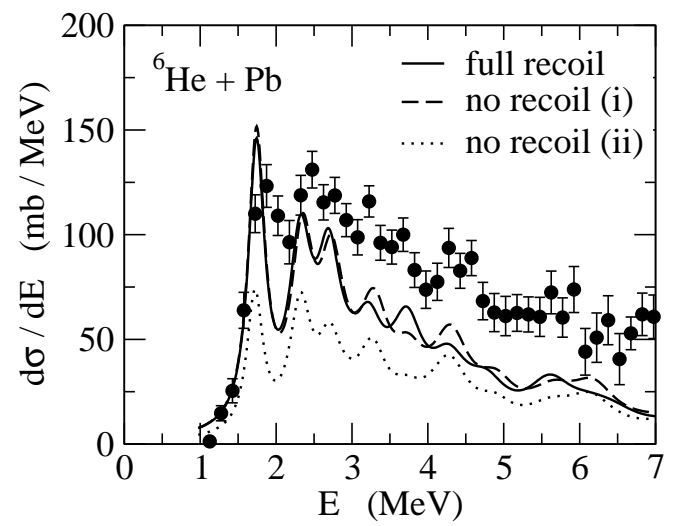

FIG. 2: Coulomb breakup cross sections for ${ }^{6} \mathrm{He}+\mathrm{Pb}$ at 240 $\mathrm{MeV} /$ nucleon. The solid line is the result of the full threebody calculations, while the dashed line is obtained by neglecting the off-diagonal component of the recoil kinetic energy in the excited states. The dotted line is obtained by neglecting the off-diagonal recoil term both in the ground and the excited states. These results are smeared with an energy dependent width of $\Gamma=0.15 \cdot \sqrt{E_{\text {rel }}} \mathrm{MeV}$. The experimental data are taken from Ref. [7].

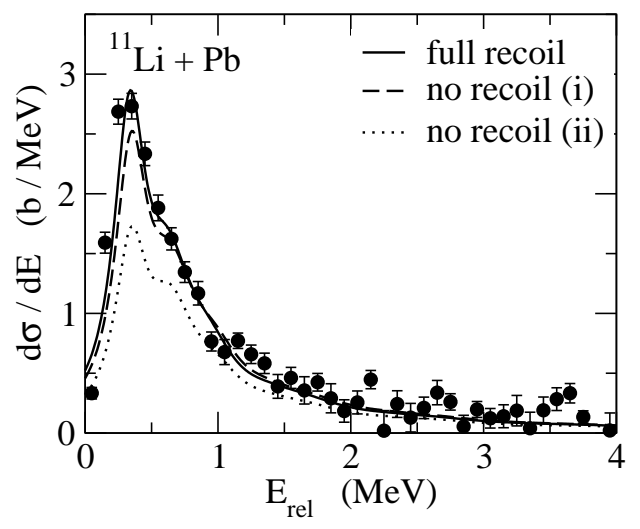

FIG. 3: Coulomb breakup cross sections for the ${ }^{11} \mathrm{Li}+\mathrm{Pb}$ at $70 \mathrm{MeV} /$ nucleon. The meaning of each line is the same as in Fig. 2. The calculated results are smeared with an energy dependent width of $\Gamma=0.25 \cdot \sqrt{E_{\text {rel }}} \mathrm{MeV}$. The experimental data are taken from Ref. [6].

We next evaluate the Coulomb breakup cross sections, especially paying attention to the recoil effect of the core nucleus. Based on the relativistic Coulomb excitation theory [17, 18], the cross sections are obtained by multiplying the virtual photon number $N_{\mathrm{E} 1}(E)$ to the $\mathrm{B}(\mathrm{E} 1)$ distribution shown in Fig. 1. The solid curves in Figs. 2 and 3 show the Coulomb breakup cross sections thus obtained for ${ }^{6} \mathrm{He}+\mathrm{Pb}$ reaction at $240 \mathrm{MeV} /$ nucleon 7 ] and ${ }^{11} \mathrm{Li}+\mathrm{Pb}$ reaction at $70 \mathrm{MeV} /$ nucleon [6], respectively. In order to facilitate the comparison with the experimental data, we smear the discretized cross sections with the Lorenzian function with an energy dependent width, $\Gamma=\alpha \cdot \sqrt{E_{\text {rel }}}$. We take $\alpha=0.15$ and $0.25 \mathrm{MeV}^{1 / 2}$ for ${ }^{6} \mathrm{He}$ and ${ }^{11} \mathrm{Li}$, respectively. We see that the experimental 


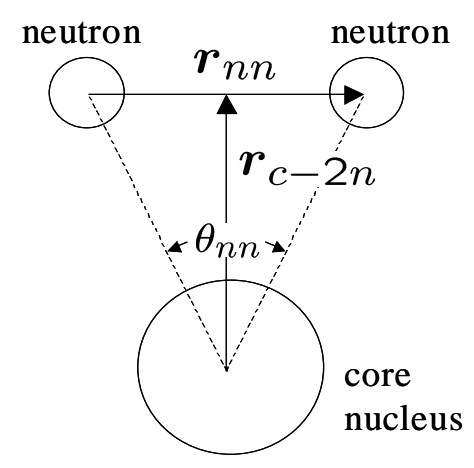

FIG. 4: Geometry of a $2 n$ halo nucleus consisting of a core nucleus and two valence neutrons.

breakup cross sections are reproduced remarkably well within the present three-body model, especially for the ${ }^{11} \mathrm{Li}$ nucleus.

One of the advantages to use the contact interaction is that the continuum response can be calculated relatively easily [9]. In the presence of the recoil kinetic energy of the core nucleus, however, this advantage disappears since the off-diagonal part of the recoil energy, $\boldsymbol{p}_{1} \cdot \boldsymbol{p}_{2} /\left(A_{c} m\right)$ (the last term in Eq. (1) of Ref. [13]) is a finite range two-body term, although the diagonal part, $\left(\boldsymbol{p}_{1}^{2}+\boldsymbol{p}_{2}^{2}\right) /\left(2 A_{c} m\right)$, can be easily included through the reduced mass. In order to examine the effect of the recoil term, Figs. 2 and 3 compare the Coulomb breakup cross sections calculated by taking into account the recoil term exactly (the solid curves) with those calculated approximately (the dashed and dotted curves). For the dashed curves, the off-diagonal component of the recoil kinetic energy is neglected in the excited $J^{\pi}=1^{-}$states, while it is fully taken into account in the ground state. It is interesting to notice that these calculations lead to similar results to the one in which the recoil term is treated exactly (the solid curves). The continuum response was obtained in this way in Ref. [10] for the ${ }^{11} \mathrm{Li}$ nucleus. The dotted curves, on the other hand, are obtained by neglecting the off-diagonal part of the recoil term both for the ground and the $J^{\pi}=1^{-}$states. For this calculation, we slightly readjust the parameters of the pairing interaction so that the ground state energy remains the same. By neglecting the recoil term in the ground state, the value for $\left\langle r_{c-2 n}^{2}\right\rangle$ decreases, from $13.2 \mathrm{fm}^{2}$ to $9.46 \mathrm{fm}^{2}$ for ${ }^{6} \mathrm{He}$ and from $26.3 \mathrm{fm}^{2}$ to $20.58 \mathrm{fm}^{2}$ for ${ }^{11} \mathrm{Li}$. Consequently, the $\mathrm{B}(\mathrm{E} 1)$ distribution as well as the breakup cross sections are largely underestimated. The fraction of the main components in the ground state wave function are also altered by neglecting the recoil term: for ${ }^{6} \mathrm{He}$, the fraction of the $\left(\mathrm{p}_{3 / 2}\right)^{2}$ component changes from 83.0 $\%$ to $90.8 \%$, and for ${ }^{11} \mathrm{Li}$, the fraction of the $\left(\mathrm{s}_{1 / 2}\right)^{2}$ component changes from $22.6 \%$ to $17.1 \%$, and the fraction of the $\left(\mathrm{p}_{1 / 2}\right)^{2}$ component from $59.1 \%$ to $65.7 \%$. These results clearly indicate that the recoil term is important for the ground state, while it has a rather small effect on the excited states.
Let us now discuss the geometry of the ${ }^{6} \mathrm{He}$ and ${ }^{11} \mathrm{Li}$ nuclei. Using the experimental value for $\left\langle r_{c-2 n}^{2}\right\rangle$ obtained from the $\mathrm{B}(\mathrm{E} 1)$ distribution, one can extract the mean opening angle between the valence neutrons once an additional information is available. In Ref. [6], Nakamura et al. have used the non-correlated calculation for B(E1) distribution given in Ref. [9] to estimate the rms distance between the core nucleus and one of the valence neutrons, and obtained the mean opening angle of $\left\langle\theta_{n n}\right\rangle=48_{-18}^{+14}$ degrees. However, this method is highly model dependent and also it is not obvious whether the non-correlated calculation is reasonable to estimate the rms distance. The mean opening angle can be extracted more directly when the rms distance between the valence neutrons, $\left\langle r_{n n}^{2}\right\rangle$, is available (see Fig. 4). This quantity is related to the matter radius and $\left\langle r_{c-2 n}^{2}\right\rangle$ in the three-body model [8, 10, 15, 19],

$$
\left\langle r_{m}^{2}\right\rangle=\frac{A_{c}}{A}\left\langle r_{m}^{2}\right\rangle_{A_{c}}+\frac{2 A_{c}}{A^{2}}\left\langle r_{c-2 n}^{2}\right\rangle+\frac{1}{2 A}\left\langle r_{n n}^{2}\right\rangle,
$$

where $A_{c}=A-2$ is the mass number of the core nucleus. The matter radii $\left\langle r_{m}^{2}\right\rangle$ can be estimated from interaction cross sections. Employing the Glauber theory in the optical limit, Tanihata et al. have obtained $\sqrt{\left\langle r_{m}^{2}\right\rangle}=1.57 \pm$ $0.04,2.48 \pm 0.03,2.32 \pm 0.02$, and $3.12 \pm 0.16 \mathrm{fm}$ for ${ }^{4} \mathrm{He}$, ${ }^{6} \mathrm{He},{ }^{9} \mathrm{Li}$, and ${ }^{11} \mathrm{Li}$, respectively [20]. Using these values, we obtain the rms neutron-neutron distance of $\sqrt{\left\langle r_{n n}^{2}\right\rangle}$ $=3.75 \pm 0.93$ and $5.50 \pm 2.24 \mathrm{fm}$ for ${ }^{6} \mathrm{He}$ and ${ }^{11} \mathrm{Li}$, respectively. Combining these values with the rms coredineutron distance, $\sqrt{\left\langle r_{c-2 n}^{2}\right\rangle}$, obtained with Eq. (2), we obtain the mean opening angle of $\left\langle\theta_{n n}\right\rangle=51.56_{-12.4}^{+11.2}$ and $56.2_{-21.3}^{+17.8}$ degrees for ${ }^{6} \mathrm{He}$ and ${ }^{11} \mathrm{Li}$, respectively. These values are comparable to the result of the three-body model calculation, $\left\langle\theta_{n n}\right\rangle=66.33$ and 65.29 degree for ${ }^{6} \mathrm{He}$ and ${ }^{11} \mathrm{Li}$, respectively [13], although the experimental values are somewhat smaller. We should remark here that it is misleading to say that the two neutrons are mostly sitting with an opening angle obtained in this way. Instead, the mean opening angle is most probably an average of a smaller and a larger correlation angles in the density distribution as has been suggested in Ref. [13].

An alternative way to extract the value for $\sqrt{\left\langle r_{n n}^{2}\right\rangle}$ has been proposed which uses the three-body correlation study in the dissociation of two neutrons in halo nuclei 12. The two neutron correlation function provides the experimental values for $\sqrt{\left\langle r_{n n}^{2}\right\rangle}$ to be $5.9 \pm 1.2$ and 6.6 $\pm 1.5 \mathrm{fm}$ for ${ }^{6} \mathrm{He},{ }^{11} \mathrm{Li}$, respectively [12]. Very recently, Bertulani and Hussein used these values to estimate the mean opening angles and obtained $\left\langle\theta_{n n}\right\rangle=83_{-10}^{+20}$ and 66 ${ }_{-18}^{+22}$ degrees for ${ }^{6} \mathrm{He}$ and ${ }^{11} \mathrm{Li}$, respectively [11]. When one adopts the presently obtained value for $\sqrt{\left\langle r_{c-2 n}^{2}\right\rangle}$ with Eq. (2) instead of those in Refs. [6, 7], one obtains $\left\langle\theta_{n n}\right\rangle=74.5_{-13.1}^{+11.2}$ and $65.2_{-13.0}^{+11.4}$ degrees for ${ }^{6} \mathrm{He}$ and ${ }^{11} \mathrm{Li}$, respectively. Notice that these values are in better agreement with the results of the three-body calculation [13], 
TABLE I: The geometry of the ${ }^{6} \mathrm{He}$ and ${ }^{11} \mathrm{Li}$ nuclei extracted from various experimental data. The mean opening angles calculated with the three-body model [13] are given in the last line for each nucleus in the table.

\begin{tabular}{c|c|cc|c}
\hline \hline nucleus & $\begin{array}{c}\sqrt{\left\langle r_{c-2 n}^{2}\right\rangle} \\
(\mathrm{fm})\end{array}$ & $\begin{array}{c}\sqrt{\left\langle r_{n n}^{2}\right\rangle} \\
(\mathrm{fm})\end{array}$ & method & $\begin{array}{c}\left\langle\theta_{n n}\right\rangle \\
(\text { degree })\end{array}$ \\
\hline${ }^{6} \mathrm{He}$ & $3.88 \pm 0.32$ & $3.75 \pm 0.93$ & (matter radii) & $51.6_{-12.4}^{+11.2}$ \\
& & $5.9 \pm 1.2$ & $(2$-correlations $)$ & $74.5_{-13.1}^{+11.2}$ \\
& & & & $66.33[13]$ \\
\hline${ }^{11} \mathrm{Li}$ & $5.15 \pm 0.33$ & $5.50 \pm 2.24$ & (matter radii) & $56.2_{-21.3}^{+17.8}$ \\
& & $6.6 \pm 1.5$ & (2n-correlations) & $65.2_{-13.0}^{+11.4}$ \\
& & & & $65.29[13]$ \\
\hline \hline
\end{tabular}

especially for the ${ }^{6} \mathrm{He}$ nucleus, as compared to the values obtained by Bertulani and Hussein. We summarize our results in Table I. One should notice that there are still large uncertainties in the empirical values of $\sqrt{\left\langle r_{n n}^{2}\right\rangle}$ and, consequently, in the average opening angles $\left\langle\theta_{n n}\right\rangle$ as listed in Table 1. It is still an open challenging problem to determine experimentally the values for $\sqrt{\left\langle r_{n n}^{2}\right\rangle}$ with higher precision.
In summary, we have used the three-body model with a density dependent contact interaction to analyze the $\mathrm{B}(\mathrm{E} 1)$ distributions as well as the Coulomb breakup cross sections of the ${ }^{6} \mathrm{He}$ and ${ }^{11} \mathrm{Li}$ nuclei. We have shown that the strong concentration of the $\mathrm{B}(\mathrm{E} 1)$ strength near the continuum threshold can be well reproduced with the present model for both the nuclei. We have also examined the recoil effect of the core nucleus on the Coulomb breakup cross sections. It is shown that the recoil effect plays an important role in the ground state while it may be neglected in the excited states. Using the calculated $\mathrm{B}(\mathrm{E} 1)$ strength, we extracted the experimental value for the rms distance between the core and the center of two neutrons, which was then converted to the mean opening angle of the two valence neutrons from the core nucleus. We have found that the mean opening angles thus obtained are in good agreement with the results of the three-body model calculation.

We thank T. Aumann and T. Nakamura for useful discussions on the experimental data of B(E1) strength distributions in the ${ }^{6} \mathrm{He}$ and ${ }^{11} \mathrm{Li}$ nuclei. This work was supported by the Japanese Ministry of Education, Culture, Sports, Science and Technology by Grant-in-Aid for Scientific Research under the program number 19740115.
[1] J.M. Blatt and V.F. Weisskopf, Theoretical Nuclear Physics, (John Wiley \& Sons, New York, 1952).

[2] C.A. Bertulani, G. Baur, and M.S. Hussein, Nucl. Phys. A526, 751 (1991).

[3] H. Sagawa, N. Takigawa, and Nguyen Van Giai, Nucl. Phys. A543, 575 (1992).

[4] M.A. Nagarajan, S.M. Lenzi, and A. Vitturi, Eur. Phys. J. A24, 63 (2005).

[5] S. Typel and G. Baur, Nucl. Phys. A759, 247 (2005).

[6] T. Nakamura et al., Phys. Rev. Lett. 96, 252502 (2006).

[7] T. Aumann et al., Phys. Rev. C59, 1252 (1999).

[8] G.F. Bertsch and H. Esbensen, Ann. Phys. (N.Y.) 209, 327 (1991).

[9] H. Esbensen and G.F. Bertsch, Nucl. Phys. A542, 310 (1992).

[10] H. Esbensen, K. Hagino, P. Mueller, and H. Sagawa, Phys. Rev. C76, 024302 (2007).

[11] C.A. Bertulani and M.S. Hussein, arXiv:0705.3998.
[12] F.M. Marques et al., Phys. Lett. B476, 219 (2000).

[13] K. Hagino and H. Sagawa, Phys. Rev. C72, 044321 (2005).

[14] K. Hagino, H. Sagawa, J. Carbonell, and P. Schuck, Phys. Rev. Lett. 99, 022506 (2007).

[15] H. Esbensen, G.F. Bertsch and K. Hencken, Phys. Rev. C56, 3054 (1999).

[16] K. Hagino and H. Sagawa, Phys. Rev. C75, 021301 (R), (2007).

[17] C.A. Bertulani and G. Baur, Phys. Rep. 163, 299 (1988); Nucl. Phys. A480, 615 (1988).

[18] A. Winther and K. Alder, Nucl. Phys. A319, 518 (1979).

[19] N. Vinh Mau and J.C. Pacheco, Nucl. Phys. A607, 163 (1996).

[20] I. Tanihata et al., Phys. Lett. B206, 592 (1988); A. Ozawa et al., Nucl. Phys. A693, 32 (2001). 